\title{
Non-contact breath sensor based on a Doppler detector
}

\author{
Z. R. Szczepaniak \& M. Łuszczyk \\ Przemystowy Instytut Telekomunikacji S.A. Warsaw, Poland
}

\begin{abstract}
Respiratory activity is an important parameter for observation of human body activity. A normal adult has a respiratory rate of $12-15$ breaths/min in normal conditions. Breath sensor based on non-contact Doppler detector allows for the measurement and detection and the absence of the breathing action, which may cause death in a few minutes. The monitoring and detection of some respiratory abnormality presents interest in certain situations (i.e. patients from intensive therapy, of newborn children and many others). The paper covers a new technical solution of the low-cost breath sensor, which consists of the microwave generator together with a resonant patch antenna. There is only one antenna for transmitting and receiving. The technical solution of the oscillator is based on a single FET transistor. The microwave oscillator may be tuned by change of the antenna dimensions. The solution presented here is designed for $2.4 \mathrm{GHz}$ (ISM band). The respiratory activity is mainly detected due to the motion of the body. The wave reflected by the moving body surface is mixed with the oscillating frequency by FET transistor junction. Filtering the low frequency signals gives a component that represents the Doppler frequency due to body surface motion. Next, it is processed with high a resolution analogue-digital converter and digitally filtered, and time-frequency analyzed as well. The processing enables the detection of the respiration rate with accuracy 1 beat $/ \mathrm{min}$ or $0.016 \mathrm{~Hz}$. Signal processing in digital domain includes removal of DC offset and out-of-band noise. Experimental results confirm the possibility of using a microwave Doppler detector to measure and analyze respiratory activity signals. This method permits to measure the breathing rate period.
\end{abstract}

Keywords: respiratory sensor, microwave Doppler detector. 


\section{Introduction}

Recently, interest in non-contact breath sensors has increased rapidly. Advances in microwave technology make it possible to design very small and simple devices for detecting vital signs. New applications of non-contact sensing of human beings have also been conceived in medical applications and as tools for detecting human beings hidden behind walls for instance, or as rescue tools for finding survivors trapped under rubble [1].

The research activities are currently focussed on the use of two different techniques for vital signs detection by means of microwave sensors: ultrawideband $(\mathrm{UWB})$ radars and continuous wave $(\mathrm{CW})$ radars.

UWB radars transmit short pulses with a pulse duration of the order of nanoseconds [6]. This type of radar, as well as CW radars, is able to detect the movement of a target by measuring the low-Doppler variation that affects the received backscattered signal. UWB radars also provide a range resolution that permits to eliminate the interfering pulses due to reflections of other targets in the background. However, that characteristic requires a fast switching time discriminator that opens the receiver when the wanted reflected pulse arrives. If the distance is changed, the delay of the time window of the discriminator must be adjusted.

CW radars are simpler systems than the UWB radars and the received signal processing is independent on the target distance [3]. But in order to measure the displacements due to breathing, other movements of the subject under observation, different from that of respiratory, should be avoided. Several CW radar transducer configurations have been developed to deal with sensitivity and detection capability. Sensors working in various frequency ranges (i.e. S-band, C-band and K-band) have been tested to adapt the transmitted wavelength to the chest movements. Quadrature direct-conversion systems or double sideband indirect-conversion systems have been carried out to resolve the null point problem, which causes an accuracy decrease related to the distance between the sensor and the chest. However, all the above-mentioned systems involve a typical transducer with transmitting and receiving parts.

\section{$2 \mathrm{CW}$ radar sensor features}

Doppler-radar motion-sensing systems typically transmit a continuous-wave (CW) signal (sometimes frequency modulated), which is reflected off a target and then demodulated in the receiver [4]. According to Doppler theory, a target with a time-varying position but a net zero velocity, will reflect the signal with its phase modulated proportionally to the time-varying target position. A stationary person's chest has a periodic movement with a zero velocity, and therefore, a CW radar with the chest as the target will receive a signal similar to the transmitted signal with its phase modulated by the time-varying chest position. Demodulating the phase will then give a signal directly proportional to the chest position, which contains information about movement due to heartbeat and respiration, from which heart and respiration rates and signatures can be 
determined [1]. Based on this principle, a noncontact heart and respiration monitor can be envisioned. By leveraging existing wireless technology, it would be possible to manufacture an inexpensive and portable device to perform such monitoring. Assuming that there is no amplitude noise, a $\mathrm{CW}$ radar typically transmits a single-tone signal:

$$
s_{n}(t)=A_{n} \cos [2 \pi f t+\phi(t)]
$$

where: $f$ - signal frequency, $\phi(\mathrm{t})$ - phase noise of the oscillator, $t$-time.

The transmitted signal reflects from body surface and returns to the receiving antenna. After amplification it is led to a phase detector. The received signal is phase-shifted and this phase shift is a function of emitted wavelength and distance between radar and monitored object. Moreover, when the surface is being radiated (e.g. chest surface) changes its position periodically $x(t)$ (with mean value equal to zero), the resulting phase shift is periodically modulated.

$$
S_{o}(t)=A_{o} \cos \left[2 \pi f t-\frac{4 \pi L}{\lambda}-\frac{4 \pi x(t)}{\lambda}+\phi\left(t-\frac{2 L}{c}\right)\right]
$$

where: $c$-speed of EM wave in vacuum, $\lambda$ - wavelength in the air

The output signal of phase detector is a function of phase difference between transmitted and received signals.

$$
S_{D}(t)=\cos \left[\theta_{0}+\frac{4 \pi L}{\lambda}+\frac{4 \pi x(t)}{\lambda}+\Delta \phi(t)\right]
$$

where: $4 \pi L / \lambda$-phase shift depending on target distance,

$\theta_{0}$ - constant phase shift resulting from signal delays in the transmit/receive circuitry (for given structure of transmitter /receiver), $\Delta \phi(t)$ - component resulting from conversion of phase noise to baseband.

The single-channel Doppler radar exhibits minima of sensitivity for specific target distances expressed as:

$$
\theta_{0}+\frac{4 \pi L}{\lambda}=n \pi
$$

\section{Microwave sensor description}

The sensor presented here has very simple structure. The main assumption is one channel operation without splitting of the received signal into the in-phase and 
quadrature channels. Typically, the sensor setup consists of microwave signal source, splitters, amplifiers, mixers and two antennas. On the other hand, there are solutions using signal generating circuitry based on IMPATT diode and rectangular waveguide resonator. Transmitting and receiving of the signals is accomplished by means of one horn antenna. The IMPATT diode forms an active circuit for oscillator and short-circuited section of rectangular waveguide plays a role of microwave resonator, and therefore a passive circuit for oscillator. The advantage of this solution is that the mixing of transmitted and received signals takes place in nonlinear junction of semiconductor diode without any additional mixer circuit. The disadvantage is that this simple one-channel circuit suffers from the nulling effect.

The solution presented here relies on the loop-type microwave oscillator. Here, the active circuit is formed with the use of microwave HEMT transistor (2, see fig. 1). The active circuit is a two-port network. The passive circuit is formed by two-port resonant circuit which simultaneously plays a role of patch antenna. Therefore, this solution is more compact and simpler than presented earlier [5]. The use of radiating element as a resonant circuit in the oscillator gives additional substantial advantage. When the distance from antenna to monitored object is decreased the object enters near field area and antenna resonant circuit starts to be tuned by presence of the object material in the field region. The oscillator should be properly designed; it means taking into account the presence of object at possible closest position to the antenna. As an effect, the "targettuned" oscillator is obtained.

For different target distance from the antenna the oscillator conditions of generation are still fulfilled. This means that for every target position oscillator generates output signal with different position-dependant frequency. For small periodic variation of position the frequency modulation will occur.

Finally, the component at intermediate frequency will appear in the active nonlinear element bias circuit (1, see fig. 1). In that way, for close range operation, there is no nulling effect due to distance between object and antenna.

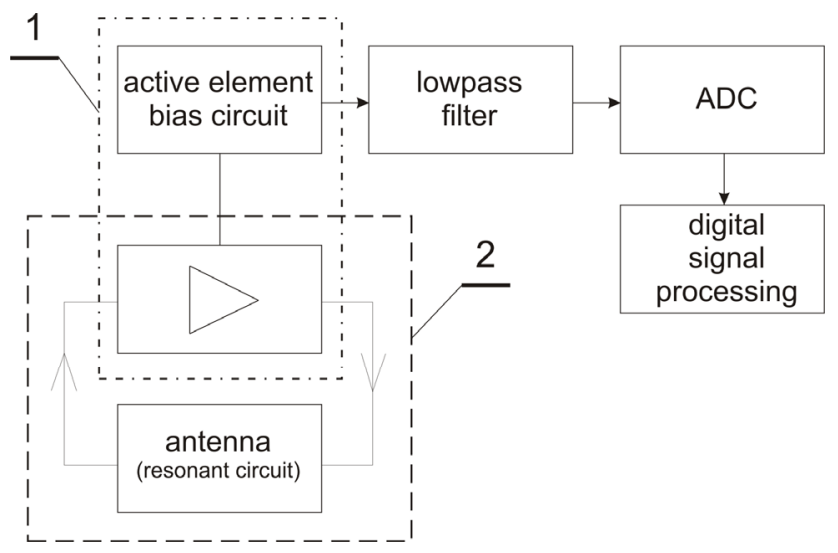

Figure 1: Microwave Doppler sensor structure. 


\section{Signal processing in Doppler sensor}

In Doppler respiration measurement signal processing is realized in analogue and digital domain. The initial part of the signal processing in discussed Doppler sensor is baseband signal conditioning. Before a signal is digitized it must be lowpass filtered to avoid out-of-band interference alias signals into baseband signal. The bit resolution of the analog-to-digital converter determines kind of preprocessing steps. 16-bit resolution converter used in measurement set-up is enough to resolve respiration signal. In Doppler sensor the dc offset is sufficiently large that the level of amplification must be set carefully not to saturate the converter. When the signal is small and noisy the dc offset must be removed before or simultaneously with amplification.

Anti-aliasing filter is required to limit a bandwidth less than half the sampled rate to avoid aliasing effect. This problem is resolved with active analog lowpass filter with cutoff frequency below Nyquist frequency (sampling frequency is typically performed at five times the signal bandwidth. In measurement setup frequency sampling is $100 \mathrm{~Hz}$. The filter is realized as Sallen-Key lowpass filter with cutoff frequency equals $25 \mathrm{~Hz}$ (fig. 2). The cutoff frequency is sufficient to limit any sampling frequency above $50 \mathrm{~Hz}[1]$.

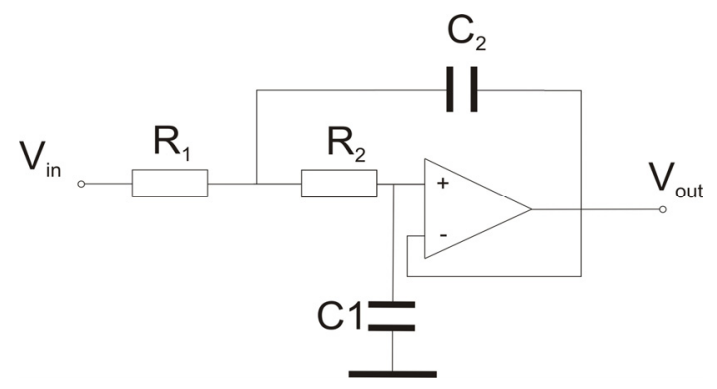

Figure 2: Anti-aliasing Sallen-key lowpass filter circuit.

The dc blocking circuit and amplification circuit is shown in figure 2. Typical signal amplitude is between 0.5 and $1 \mathrm{mV}$. To amplify the signal to full scale of $\mathrm{ADC}(0-5 \mathrm{~V}$ or \pm 5 typically) the amplification of about $80 \mathrm{~dB}$ is required. Due to dc offset, gain below is $40 \mathrm{~dB}$ suitable. The dc-block circuit efficiency depends on Doppler sensor bias stability.

In the Doppler sensor the respiration and heartbeat signal are superimposed on each other. The chest move amplitude due to breath is much bigger than this of the heart beating. The respiration signal is typically about 100 times greater than that of the signal due to the heartbeat. The respiration signal can be detected and processed without filtering but detection of heartbeat signal requires filtering.

Digital signal processing (DSP) includes digitization process and digital filtering. Digitization in discussed system is $100 \mathrm{~Hz}$ and has 16 -bit resolution. Signal conditioning steps include removal of $\mathrm{dc}$ offset and filtering which removes out-of band noise and separates breath signal and other signal (i.e. heartbeat signal or backscatter signal or clutter signal). 


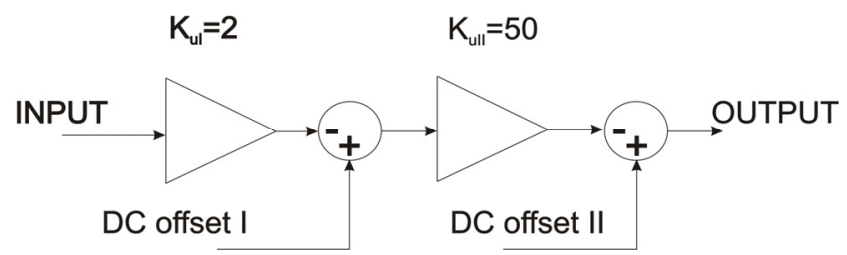

Figure 3: $\quad$ DC offset reduction circuit.

The DSP is based on fact that required respiration signal and other signals are separated in frequency domain. Resting respiration rate is between 0.15 and $0.4 \mathrm{~Hz}$ what corresponds to 9 and 24 breaths per minute [2]. Heart rate is between 0.83 and $1.5 \mathrm{~Hz}$ what corresponds to 50 and 90 beats per minute. This means that the breath signal can be isolated by a lowpass filter. A sample of respiration and heart signals measured with a microwave Doppler sensor are shown in fig. 4 and fig. 5 .

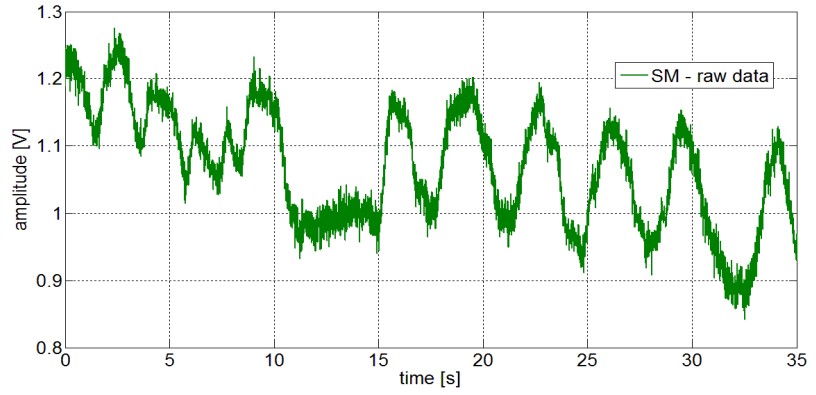

Figure 4: $\quad$ Respiration raw signal measured at $20 \mathrm{~cm}$ distance (chest).

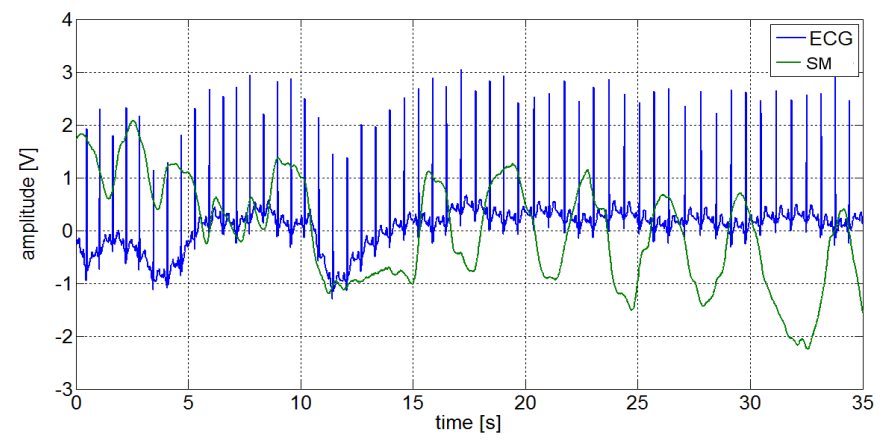

Figure 5: Respiration signal after the lowpass filter with $1 \mathrm{~Hz}$ cut-off frequency (SM) and reference signal ECG (variation of isoline).

Respiration and heart signals are separated with digital filters. Filter isolating heartbeat must attenuate the respiration signal at last $50 \mathrm{~dB}$. Signal processing with finite impulse response (FIR) is presented. The FIR filters use current and past input samples. The number of coefficients ant its values determine the 
filter's properties in frequency and time domain (i.e. cut-off frequency, the steepness of transition between the passband and stopband, group delay and how long the filter has an output signal given a step at the input). The phase shift of an FIR filter is linear within passband and group delay is constant and its value depends on filter order. To separate heartbeat and respiration signal FIR 250order Kaiser filter with parameter $\beta=2$ with 3 second group delay is used.

The Fourier Transform is used to determine the frequency characteristics of respiration signal (fig. 6). When the frequency characteristics varies in time (non-stationary signals) what is typical for physiological signals short-time Fourier transform should be used for analysis.

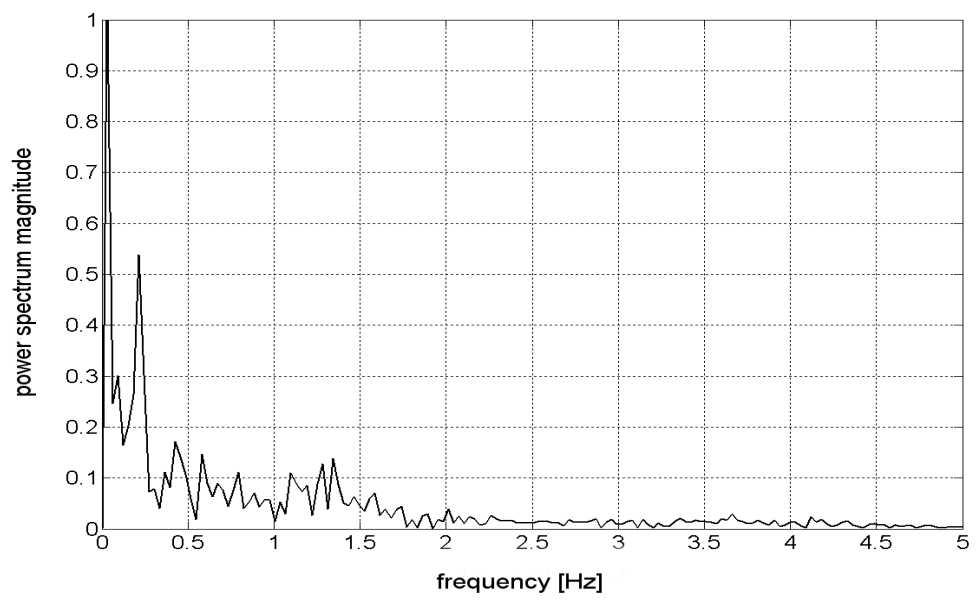

Figure 6: Respiration signal in frequency domain.

For heart monitoring the heartbeat signal must be isolated by filtering with highpass filter. The Doppler sensor signal filtered with 400 -order Kaiser $(\beta=2.5)$ filter is shown in fig. 7.

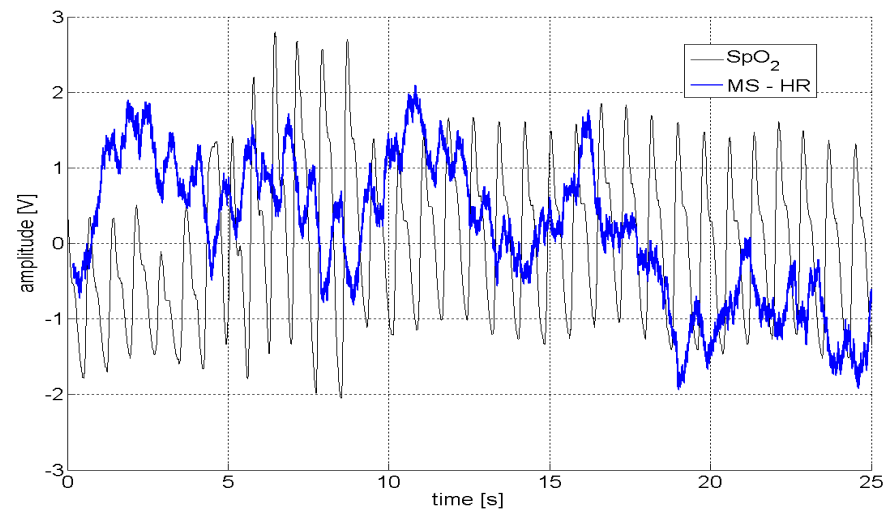

Figure 7: Heartbeat signal measured at $20 \mathrm{~cm}$ (chest MS-HR) with reference from pulse oximeter $\left(\mathrm{SpO}_{2}\right)$ 


\section{Conclusions}

A low cost microwave sensor for human breath and heart rate activity is presented. The circuit concept is based on a loop-type microwave oscillator with resonant circuit acting as antenna. The oscillator is designed to be frequencytuned by the change of target distance from the antenna. This feature allows avoidance of the nulling effect. The sensor structure is very compact. The concept has been verified by the measurement of the model sensor with the use ECG and pulse oximeter as the reference.

Proper detection of breath action has been obtained form the distance of $20 \mathrm{~cm}$ from the chest surface.

This kind of sensors may be used in distributed systems for monitoring the patient activity in the field of health-care or to verify the condition of machine operator (pilot, drive, etc.).

\section{References}

[1] Droitcour A., Lubecke V., Lin J.C., Boric-Lubecke O.: A microwave radio for Doppler radar sensing of vital signs. 2001 IEEE MTT-S Int. Microwave Symp. Dig., 2001, vol. 1, pp. 175-178.

[2] Droitcour A. D., Boric-Lubecke O., Lubecke V. M., Lin J., Kovacs G.: Range correlation and I/Q performance benefits in single-chip silicon Doppler radars for noncontact cardiopulmonary monitoring. IEEE Transactions on Microwave Theory and Techniques, vol. 52, no. 3, pp. 838848,2004

[3] Lin J.C.: Microwave sensing of physiological movement and volume change: a review. Bioelectromagnetics, vol. 13, no. 6, pp. 557-565, 1992

[4] Ichapurapu R.: A 2.4GHz Non-Contact Biosensor System for Continuous Vital-Signs Monitoring, Proc. WAMICON 2009

[5] Baltag O.: Microwaves Doppler Transducer for Noninvasive Monitoring of the Cardiorespiratory Activity, IEEE Transactions On Magnetics, vol. 44, No. 11, November 2008

[6] Immoreev I.J.: Practical Application of Ultra-Wideband Radars. International Conference "Ultra Wideband and Ultra Short Impulse Signals" (UWBUSIS'06), 18-22 September, 2006, Sevastopol, Ukraine. 